\title{
OPTIMAL LOCATION PROBLEM FOR THE INSTALLATION OF POWER FLOW CONTROLLER
}

\author{
Takayuki Shiina ${ }^{1 *}$, Jun Imaizumi ${ }^{2}$, Susumu Morito ${ }^{1}$ and Chunhui $\mathrm{Xu}^{3}$
}

Received March 14, 2017 / Accepted October 23, 2017

\begin{abstract}
In power delivery systems, the use of dispersed generation and security control to improve network utilization requires the optimal use of system control devices. The installation of loop controller allows the distribution system to operate in a loop configuration, achieving effective management of voltage and power flow. In the investment planning process, it is important to identify the optimal location and installed capacity of the equipment such that all operational constraints are satisfied. This paper presents a method for identifying the optimal location and capacity with the minimum installation cost. Our novel approach uses an economic model that considers the fixed costs. A slope scaling procedure is presented, and its efficiency is demonstrated using numerical experiments.
\end{abstract}

Keywords: optimization, linear approximation, stochastic programming, power system, optimal power flow, loop controller.

\section{INTRODUCTION}

In the electric power industry, many issues are dealt with as mathematical programming problems. Representative integer programming and combinatorial optimization problems include the unit commitment problem (Shiina \& Birge, 2004; Shiina \& Watanabe, 2004) and the power generation planning problem (Shiina \& Birge, 2003). Discussions of the application of stochastic programming methods to the electricity industry can be found in works by Ruszczyński \& Shapiro (2003) and by Shapiro et al. (2009). For these problems, the branch-and-bound methods are used in which discrete variables are enumerated, techniques that relax the constraints, or approximation methods such as local search. Solution methods using a discrete structure for the problem are most commonly used. Until recently, it has been extremely difficult to solve largescale combinatorial optimization problems, but the development of mathematical programming methods has provided techniques that allow approximate solutions to be found effectively. These

\footnotetext{
*Corresponding author.

${ }^{1}$ Waseda University, 3-4-1 Okubo, Shinjuku, Tokyo 169-8555 Japan. E-mail: tshiina@waseda.jp

2 Toyo University, 5-8-20 Hakusan, Bunkyo, Tokyo 112-8606 Japan. E-mail: jun@ toyo.jp

${ }^{3}$ Chiba Institute of Technology, 2-17-1 Tsudanuma, Narashino 275-0016 Japan. E-mail: joh.haruki@ gmail.com
} 
approximation methods are referred to collectively as metaheuristics. If the local search method is to function effectively, then it is important that the set of feasible solutions be identified quickly.

In contrast, the basic economic power dispatch problem is a nonlinear programming problem. In the case of convex nonlinear programming problems, solution methods that solve large-scale problems effectively have been demonstrated (Bazaraa et al., 1993). Shiina (1999) adressed a convex programming problem that considered the uncertainty in demand in a power supply. However, the problem becomes very challenging when convex programming cannot be applied. In calculating the optimal power flow (OPF) (Wood \& Wollenburg, 1996; Zhu, 2015), because the flow equation is described as a nonlinear equality constraint, the problem becomes one of nonlinear optimization with a feasible set that is not convex. In this case, a feasible solution may not be obtainable. In recent years, solutions based on the semidefinite programming have been studied (Bai et al., 2008; Molzahn et al., 2013). However, implementation using these solution method is not always easy to introduce. A simple method using software that is easy to obtain is desired.

The problem considered in the present paper is one of optimization under combinatorial conditions that represent the location of the equipment and the installation of a loop controller (LPC) to control the power flow, in addition to the calculation of the OPF. The LPC operates the current distribution network in a loop configuration. It can be installed between two specific points on the network and its capacity must also be decided. From the viewpoint of suppressing the cost, the LPC can effectively utilize existing facilities. In addition, since it has a very simple structure, it is possible to introduce various types of power supply and deal with fluctuations in demand flexibly. The installation of equipment is formulated as an integer programming problem or a combinatorial optimization problem, but because the calculation of flow is a non-convex nonlinear programming problem, a solution is difficult to find. This problem takes the power demand as an input condition and determines the installation that minimizes costs under the operational constraints of the network. The network operational constraints to be considered here are the voltage operating range and the line thermal capacity. However, when optimizing large-scale systems or considering flow constraints in multiple scenarios, the number of variables and constraints in the optimization problem becomes huge, and a more efficient optimization technique is required. A conventional technique uses a two-stage approach that mixes combinatorial optimization and nonlinear optimization, in which the search for a location and the determination of the capacity of the equipment are treated separatedly. When this technique is applied, there are many cases in a local search for the installation location in which no feasible flow exists, which makes it difficult for an efficient search to be conducted. In this study, we investigated a new technique in which linearly approximation is made of the installation cost, without relying on a local search. This linear approximation method called the dynamic slope scaling procedure (Kim \& Pardalos, $1999 ; 2000)$, and has the advantage that a feasible power flow can always be obtained. Since this method approximates an objective function that includes a fixed cost, it does not include a $0-1$ variable representing the installation, and so always retrieves a feasible value for the location and power flow. 
The rest of the paper is organized as follows. In Section 2, the problem is formulated. In section 3, the technique based on local search is discussed and the principle for designing a solution for the optimal installation is described. A novel algorithm based on linear approximation is then demonstrated. In Section 4, the technique is applied to the optimization of a full-scale system model. Both single scenario and multiple scenario optimizations are considered, and the effectiveness of our novel technique is demonstrated. We summarize our results in Section 5.

\section{FORMULATION OF THE OPTIMAL INSTALLATION PROBLEM}

The power system to be considered is represented by a network $N=(V, E)$, where $V$ is a node set and $E$ is the arc set, consisting of node pairs $(i, j) \in E$ such that there exists an arc between each pair with nodes $i, j$ as its ends. In the power system, a piece of electrical equipment referred to as a bus corresponds to a node on the network, and factors such as the transformer and transmission lines correspond to the arcs. The power flow through each piece of equipment in the network is called the flow.

In this paper, we consider the problem of calculating an OPF that takes into account the installation of the equipment. In order to maintain the voltage in the system at a suitable level, it is necessary to determine the location of the installation and its capacity. The collection of nodes $(i, j)$ at which the equipment can be installed, is the set $A$ of $(i, j)(i, j \in V)$, and is given as follows.

$$
A=\{(i, j) \mid \text { the equipment can be installed between nodes } i \text { and } j\}
$$

Here, in relation to set $A$, we assume that $A \cap E=\emptyset$. In other words, the equipment is not installed on the existing arcs in the network, rather we define as $A$ the collection of node pairs that are candidate locations. As we must take into account both the power flowing out of the LPC and that flowing in, the direction of $(i, j) \in A$ must be considered. When power flows from node $i$ into the equipment, we call $i$ the start node in the collection of installation nodes $(i, j)$, and when the power flows out from the equipment to node $j$, we call node $j$ the end node. The sets of start nodes and end nodes in the collection of installation nodes are designated, $\mathrm{V}^{+}$and $\mathrm{V}^{-}$, respectively, and defined as follows.

$$
\begin{aligned}
& V^{+}=\{i \in V \mid \exists(i, j) \in A, \text { power flows from node } i \text { into the equipment }\} \\
& V^{-}=\{j \in V \mid \exists(i, j) \in A, \text { power flows from the equipment into node } j\}
\end{aligned}
$$

If we remove from set $V$ the collection of nodes belonging to the set of start nodes $V^{+}$and the set of end nodes $V^{-}$belonging to set $A$, the remaining set of nodes is $\bar{V}$ and we define $\bar{V}=V \backslash\left\{V^{+} \cup V^{-}\right\}$. There is no intersection between the LPC and the nodes belonging to set $\bar{V}$. The values of the current flowing in and out of each bus, and the active and reactive power, can be calculated using the value of the voltage variable. In exceptional cases, $\bar{V}$ includes one slack bus, $N_{0} \in \bar{V}$; in the slack bus, the voltage value is given and the active and reactive power values are treated as variables. 
The binary variables relating to the installation location are determined as follows. If the equipment is installed at pair $(i, j) \in A$, then $x_{i j}=1$; if no equipment is installed, then $x_{i j}=0$. The capacity of the equipment to be installed at pair $(i, j) \in A$ is defined as variable $y_{i j}$. The objective function is the installation cost, $\alpha_{i j}$ represents the fixed costs for installation at node pair $(i, j)$, and $\beta_{i j}$ represents the variable costs per unit capacity of the equipment. The objective function is given as follows.

$$
\min \sum_{(i, j) \in A}\left(\alpha_{i j} x_{i j}+\beta_{i j} y_{i j}\right)
$$

Next, we present the constraints on the optimal installation problem. Here, $j$ is the imaginary unit. The active and reactive power of bus $i \in V$ are defined as variables $P_{i}, Q_{i}$, respectively. The active and reactive power generated at bus $i$ is given by $P G_{i}$ and $Q G_{i}$, and the load active and reactive power are given by $P L_{i}$ and $Q L_{i}$, respectively. In this case, we exclude any slack buses, and for each bus $i \in \bar{V} \backslash\left\{N_{0}\right\}$ at which the LPC is not installed, the following constraint (5) must be satisfied.

$$
P_{i}+\mathrm{j} Q_{i}=P G_{i}-P L_{i}+\mathrm{j}\left(Q G_{i}-Q L_{i}\right), i \in \bar{V} \backslash\left\{N_{0}\right\}
$$

At the slack bus $N_{0} \in \bar{V}$, no load exists and the constraint (6) must be satisfied.

$$
P_{N_{0}}+\mathrm{j} Q_{N_{0}}=P G_{N_{0}}+\mathrm{j} Q G_{N_{0}}
$$

At the slack bus, the values of $P_{N_{0}}, Q_{N_{0}}$ are given and in their place $P G_{N_{0}}, Q G_{N_{0}}$ are defined as variables. If a pair $(i, j) \in A$ is a candidate for the installation location, the active power and reactive power flowing into the equipment are represented by variables $P_{i}^{L P C}$ and $Q_{i}^{L P C}$, and the active power and reactive power flowing from the equipment into bus $j$ are represented by variables $P_{j}^{L P C}$ and $Q_{j}^{L P C}$. At the start node $i \in V^{+}$in the collection of pairs $(i, j) \in A$ that are candidates for the installation, the constraint (7) must be satisfied.

$$
\begin{aligned}
P_{i}+\mathrm{j} Q_{i}= & P G_{i}-P L_{i}+\mathrm{j}\left(Q G_{i}-Q L_{i}\right)-x_{i j}\left(P_{i}^{L P C}+\mathrm{j} Q_{i}^{L P C}\right), \\
& i \in V^{+},(i, j) \in A
\end{aligned}
$$

At the end node $i \in V^{-}$in the collection of pairs $(i, j) \in A$ that are candidates for the installation, the constraint (8) must be satisfied.

$$
\begin{aligned}
P_{i}+\mathrm{j} Q_{i}= & P G_{i}-P L_{i}+\mathrm{j}\left(Q G_{i}-Q L_{i}\right)+x_{i j}\left(P_{i}^{L P C}+\mathrm{j} Q_{i}^{L P C}\right), \\
& i \in V^{-},(i, j) \in A
\end{aligned}
$$

Here, the values of the active power flowing into the equipment and the active power flowing out from the equipment must be equal.

$$
P_{i}^{L P C}=P_{j}^{L P C},(i, j) \in A
$$

Care should be taken to ensure that there are no constraints on the reactive power flowing into and out of the LPC. 
Next, we represent the variable $V_{i}$ that indicates the voltage at bus $i$ in Cartesian coordinates as $V_{i}=e_{i}+\mathrm{j} f_{i}$, where $e_{i}$ and $f_{i}$ are variables corresponding to the real and imaginary parts of the voltage, respectively. The admittance of $\operatorname{arc}(i, j)$, namely $G_{i j}+\mathrm{j} B_{i j}$ is given. The current $I_{i}$ flowing into the network from bus $i$ can be represented by (10).

$$
I_{i}=\sum_{k=1}^{N}\left(G_{i k}+\mathrm{j} B_{i k}\right)\left(e_{i}+\mathrm{j} f_{i}\right)
$$

From this, we can represent the relation between the voltage at each bus $i \in \bar{V}$ and the active and reactive power $P_{i}, Q_{i}$ by (11), where $\bar{I}_{i}$ represents the complex conjugate of $I_{i}$.

$$
P_{i}+\mathrm{j} Q_{i}=V_{i} \bar{I}_{i}
$$

By taking the real and complex components of equation (11), we obtain the following relations.

$$
\begin{aligned}
P_{i} & =e_{i} \sum_{k=1}^{N}\left(G_{i k} e_{k}-B_{i k} f_{k}\right)+f_{i} \sum_{k=1}^{N}\left(B_{i k} e_{k}+G_{i k} f_{k}\right) \\
Q_{i} & =-e_{i} \sum_{k=1}^{N}\left(B_{i k} e_{k}+G_{i k} f_{k}\right)+f_{i} \sum_{k=1}^{N}\left(G_{i k} e_{k}-B_{i k} f_{k}\right)
\end{aligned}
$$

Using relations (12) and (13), we can represent (5) by (14) and (15) for $i \in \bar{V} \backslash N_{0}$.

$$
\begin{aligned}
e_{i} \sum_{k=1}^{N}\left(G_{i k} e_{k}-B_{i k} f_{k}\right)+f_{i} \sum_{k=1}^{N}\left(B_{i k} e_{k}+G_{i k} f_{k}\right)-P G_{i}+P L_{i} & =0 \\
-e_{i} \sum_{k=1}^{N}\left(B_{i k} e_{k}+G_{i k} f_{k}\right)+f_{i} \sum_{k=1}^{N}\left(G_{i k} e_{k}-B_{i k} f_{k}\right)-Q G_{i}+Q L_{i} & =0
\end{aligned}
$$

In the same way, using relations (12) and (13), we can represent (6) by (16) and (17), where $e_{N_{0}}$ and $f_{N_{0}}$ at the slack bus are given as constants, whereas $P G_{N_{0}}$ and $Q G_{N_{0}}$ are defined as variables.

$$
\begin{array}{r}
e_{N_{0}} \sum_{k=1}^{N}\left(G_{N_{0} k} e_{k}-B_{N_{0} k} f_{k}\right)+f_{N_{0}} \sum_{k=1}^{N}\left(B_{N_{0} k} e_{k}+G_{N_{0} k} f_{k}\right)-P G_{N_{0}}=0 \\
-e_{N_{0}} \sum_{k=1}^{N}\left(B_{N_{0} k} e_{k}+G_{N_{0} k} f_{k}\right)+f_{N_{0}} \sum_{k=1}^{N}\left(G_{N_{0} k} e_{k}-B_{N_{0} k} f_{k}\right)-Q G_{N_{0}}=0
\end{array}
$$

At the start node in the collection of pairs $(i, j) \in A$ that are candidates for the installation location, using relations (12) and (13), we can represent (7) by (18) and (19) for $i \in V^{+}$, $(i, j) \in A$.

$$
\begin{array}{r}
e_{i} \sum_{k=1}^{N}\left(G_{i k} e_{k}-B_{i k} f_{k}\right)+f_{i} \sum_{k=1}^{N}\left(B_{i k} e_{k}+G_{i k} f_{k}\right)-P G_{i}+P L_{i}+x_{i j} P_{i}^{L P C}=0 \\
-e_{i} \sum_{k=1}^{N}\left(B_{i k} e_{k}+G_{i k} f_{k}\right)+f_{i} \sum_{k=1}^{N}\left(G_{i k} e_{k}-B_{i k} f_{k}\right)-Q G_{i}+Q L_{i}+x_{i j} Q_{i}^{L P C}=0
\end{array}
$$


At the end node in the collection of pairs $(i, j) \in A$ that are candidates for the installation location, using relations (12) and (13), we can represent (8) by (20) and (21) for $i \in V^{-}$, $(i, j) \in A$.

$$
\begin{gathered}
e_{j} \sum_{k=1}^{N}\left(G_{j k} e_{k}-B_{j k} f_{k}\right)+f_{j} \sum_{k=1}^{N}\left(B_{j k} e_{k}+G_{j k} f_{k}\right)-P G_{j}+P L_{j}-x_{i j} P_{j}^{L P C}=0 \\
-e_{j} \sum_{k=1}^{N}\left(B_{j k} e_{k}+G_{j k} f_{k}\right)+f_{j} \sum_{k=1}^{N}\left(G_{j k} e_{k}-B_{j k} f_{k}\right)-Q G_{j}+Q L_{j}-x_{i j} Q_{j}^{L P C}=0
\end{gathered}
$$

The above constraints are concerned with power flow. The voltage of each bus $i$ must also satisfy inequality (22), where $V_{\max }, V_{\min }$ are given constants.

$$
V_{\min } \leq \sqrt{e_{i}^{2}+f_{i}^{2}} \leq V_{\max }, \quad i \in V
$$

For the current flowing through each $\operatorname{arc}(i, j) \in E$, an upper bound is given as (23), where $I_{\max }$ is a given constant.

$$
\left\{G_{i j}\left(e_{i}-e_{j}\right)-B_{i j}\left(f_{i}-f_{j}\right)\right\}^{2}+\left\{G_{i j}\left(f_{i}-f_{j}\right)+B_{i j}\left(e_{i}-e_{j}\right)\right\}^{2} \leq\left(I_{\max }\right)^{2}
$$

On the pairs of nodes that are candidate installation locations, the capacity $y_{i j}$ must be determined such that inequalities (24) and (25) are satisfied.

$$
\begin{aligned}
& y_{i j}^{2} \geq\left(P_{i}^{L P C}\right)^{2}+\left(Q_{i}^{L P C}\right)^{2},(i, j) \in A \\
& y_{i j}^{2} \geq\left(P_{j}^{L P C}\right)^{2}+\left(Q_{j}^{L P C}\right)^{2},(i, j) \in A
\end{aligned}
$$

Putting these together, we can formulate the mathematical programming problem as a large-scale non-convex mixed $0-1$ integer programming problem, shown here as (LPC-installation).

$$
\begin{array}{ll}
\text { (LPC-installation): } \min & \sum_{(i, j) \in A}\left(\alpha_{i j} x_{i j}+\beta_{i j} y_{i j}\right) \\
\text { subject to } & (14),(15), i \in \bar{V} \backslash\left\{N_{0}\right\} \\
& (16),(17) \\
& (9),(i, j) \in A \\
& (18),(19), i \in V^{+},(i, j) \in A \\
& (20),(21), i \in V^{-},(i, j) \in A \\
& (22), i \in V \\
& (23),(i, j), \in E \\
& (24),(25),(i, j) \in A \\
& x_{i j} \in\{0,1\},(i, j) \in A \\
& y_{i j} \geq 0,(i, j) \in A
\end{array}
$$

The present model can be used to analyze multiple scenario constraints by introducing variables for each scenario. Defining all variables exept $x$ and $y$ as stochastic makes this problem a stochastic programming problem. 


\section{SOLUTION ALGORITHM}

In this section, we demonstrate a solution using linear approximation. For a network flow problem with fixed costs, Kim \& Pardalos (1999) presented a linear approximation of the objective function, called slope scaling. This method restricts the range that the variables can take and adjustments are made at each iteration (Kim \& Pardalos, 2000). We have applied a similar linear approximation method to the optimal installation problem.

We first outline of the linear approximation method. We consider the design problem of a network with fixed costs. The network $N$ is represented as $N=(V, E)$. Sets $V$ and $E$ represent the nodes on the network and the arc set, respectively. The demand at each node $i \in V$ in set $V$ is $b_{i}$ and the column vector in which the $i$-th element is $b_{i}$ is $b \in \Re^{|V|}$. The upper bound on the flow at $\operatorname{arc}(i, j) \in E, i, j \in V$ is $u_{i j}$. The incidence matrix for the network is $A \in \mathfrak{R}^{|V| \times|E|}$. The variables are the flow on $\operatorname{arc}(i, j), v_{i j} \geq 0$ and $x_{i j} \in\{0,1\}$, which is the decision variable to include arc $(i, j)$ in the solution. The objective function is the sum of the costs in relation to the flow in each arc and the fixed costs when the flow is positive. For the case in which the fixed cost $f_{i j}$ is applied when the flow $v_{i j}$ is a positive value, and the cost per flow in $\operatorname{arc}(i, j)$ is $c_{i j}$, the following problem (FCNFP) can be formulated.

$$
\begin{aligned}
\text { (FCNFP): } \min & c v+f x \\
\text { subject to } & A v=b \\
& 0 \leq v_{i j} \leq u_{i j} x_{i j},(i, j) \in E \\
& x_{i j} \in\{0,1\},(i, j) \in E
\end{aligned}
$$

This is a mixed $0-1$ integer programming problem. If the $0-1$ variables were not present, the problem would be one of the the minimium cost flow problems, and a solution could be determined effectively. The linear approximation method solves the approximate linear programming problems obtained by adjusting the cost of the original mixed integer programming problem with fixed costs. The integer program involving variables $v$ and $x$ of (FCNFP) is replaced by an approximate problem involving only variable $v$ (FCNFP-LP). If a feasible solution $(\bar{v}, \bar{x})$ to the problem (FCNFP) is given, then the objective function value in the feasible solution, $\bar{v}$ of the approximate linear programming problem (FCNFP-LP), matches the objective function value of the original problem. Taking $c^{0}=c$, we can define the following subproblem (FCNFP-LP) at iteration $k$.

$$
\begin{aligned}
\text { (FCNFP-LP): } \min & c^{k} v \\
\text { subject to } & A v=b \\
& 0 \leq v_{i j} \leq u_{i j},(i, j) \in E
\end{aligned}
$$

In the optimal solution $v^{k}$ of (FCNFP-LP), if for $(i, j)$ such that $v_{i j}^{k}>0$ we take $x_{i j}^{k}=1$, and for $(i, j)$ such that $v_{i j}^{k}=0$ we take $x_{i j}^{k}=0$, then $\left(v^{k}, x^{k}\right)$ is the feasible solution for (FCNFP). To make the objective function value at $v=v^{k}$ for problem (FCNFP-LP) match the objective function value at $(v, x)=\left(v^{k}, x^{k}\right)$ in problem (FCNFP), we set $c_{i j}^{k+1}$ as follows, where $k$ is the current number of iterations.

$$
c_{i j}^{k+1}= \begin{cases}c_{i j}+f_{i j} / v_{i j}^{k} & \text { if } v_{i j}^{k}>0 \\ c_{i j}^{k} & \text { if } v_{i j}^{k}=0\end{cases}
$$


In this case, it can be seen from the following equations that the objective function value at solution $v=v^{k}$ for (FCNFP-LP) matches the objective function value at solution $(v, x)=$ $\left(v^{k}, x^{k}\right)$ in (FCNFP), as shown in Figure 1.

$$
\begin{aligned}
\sum_{(i, j) \in E} c_{i j}^{k+1} v_{i j}^{k} & =\sum_{(i, j) \in E, v_{i j}>0} c_{i j}^{k+1} v_{i j}^{k} \\
& =\sum_{(i, j) \in E, v_{i j}>0}\left(c_{i j}+f_{i j} / v_{i j}^{k}\right) v_{i j}^{k} \\
& =\sum_{(i, j) \in E, v_{i j}>0}\left(c_{i j} v_{i j}^{k}+f_{i j}\right)
\end{aligned}
$$

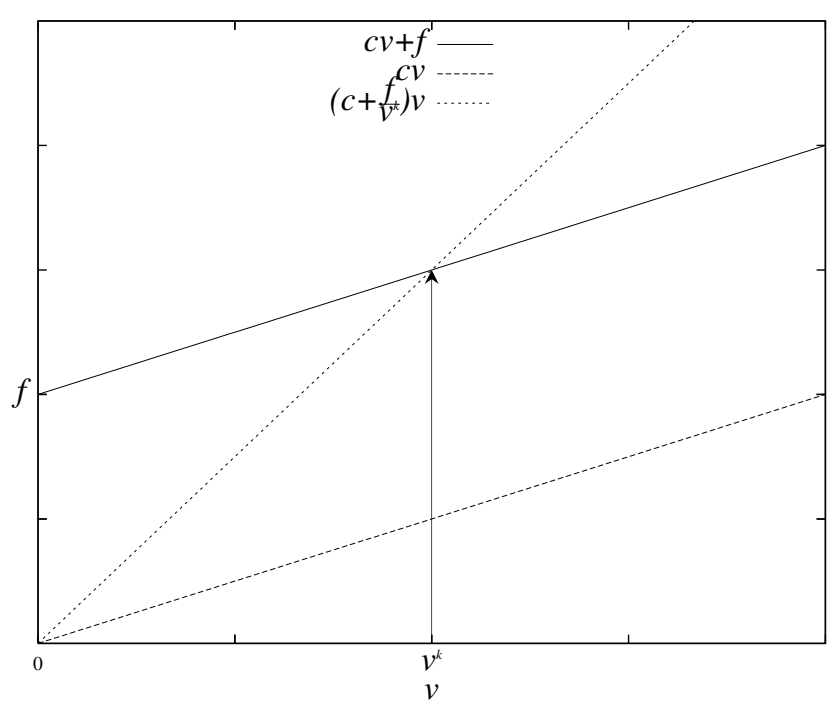

Figure 1 - Slope scaling.

The linear approximation method is applied to solve (LPC-installation). Here, we fix all $x_{i j}=$ $1,(i, j) \in A$, and solve the next problem (LPC-installation-slope) in which the cost in the objective function is adjusted.

$$
\begin{aligned}
\text { (LPC-installation-slope): } \min & \sum_{(i, j) \in A} \beta_{i j}^{k} y_{i j} \\
\text { subject to } & (14),(15), i \in \bar{V} \backslash\left\{N_{0}\right\} \\
& (16),(17) \\
& (18),(19), i \in V^{+},(i, j) \in A \\
& (20),(21), i \in V^{-},(i, j) \in A \\
& (9),(i, j) \in A \\
& (22), i \in V \\
& (23),(i, j), \in E \\
& (24),(25),(i, j) \in A \\
& x_{i j}=1, y_{i j} \geq 0,(i, j) \in A
\end{aligned}
$$


Figure 2 shows the method by which LPC installation problem is solved. The advantage of this method is that a feasible solution is always obtained. As there is no variable representing the installation location, a search for the location is not conducted. By approximating the cost function dynamically, the same cost as the conventional location cost is obtained. It is difficult to prove the convergence of the algorithm theoretically. According to Kim \& Pardalos (1999, 2000), this algorithm is a quite efficient heuristic approach for solving concave piecewise linear network flow problems. The numerical experiments show its performance is very stable.

Figure 2 - Algorithm for LPC installation.

- Step 0. (Initial setting): We take the number of iterations $k=0, \beta^{k}=\beta . \epsilon>0$ is given.

- Step 1. (Final decision condition): If $k \geq 1$, then we halt when $\sum_{(i, j) \in \mathcal{A}}\left(y_{i j}^{k}-y_{i j}^{k-1}\right)<\epsilon$. The solution $y_{i j}^{k}$ finally obtained, is the LPC capacity, when $y_{i j}^{k}>0, x_{i j}=1$ and in all other cases $x_{i j}=0$, the solution for (LPC-installation) is obtained.

- Step 2. (Calculation of (FCNFP-LP)): Problem (LPC-installation-slope)is solved, the solution $y=y^{k}$ is obtained.

- Step 3. (Update costs):

$$
\beta_{i j}^{k+1}= \begin{cases}\beta_{i j}+\alpha_{i j} / y_{i j}^{k} & \text { if } y_{i j}^{k}>0 \\ \beta_{i j}^{k} & \text { if } y_{i j}^{k}=0\end{cases}
$$

- Step 4. (Update number of iterations): Let $k=k+1$ and return to Step 1.

\section{NUMERICAL EXAMPLE USING A FULL-SCALE MODEL SYSTEM}

\subsection{Comparison between local search and the linear approximation method}

We used a full-system-scale model of a power distribution system to demonstrate the applicability of this method to the optimization of the installation location and capacity. Figure 3 shows the model used, representing a $6.6 \mathrm{kV}$ system supplying power from distribution substations at seven locations, for a standard demand scale of $250 \mathrm{MW}$ per single secondary substation $(154 / 66 \mathrm{kV})$. We assumed an urban neighborhood with an approximate area of $15 \mathrm{~km} \times 15 \mathrm{~km}$, based on the impedance value set at each feeder. The following conditions are used to model the $6.6 \mathrm{kV}$ feeder structure and load.

- One distribution substation has a three-bank (20MVA*3) structure, with four feeders coming from each transformer bank.

- There is no supply from the same transformer bank into adjacent feeders.

- One feeder is represented as a six-node circuit with two arcs branching off. The distribution line inductance is $1.2 \mathrm{mH} / \mathrm{km}$. 
- It is possible to loop the end nodes of each feeder (three nodes per feeder) with the adjacent feeder.

- The above feeder structure is common to all seven distribution substations.

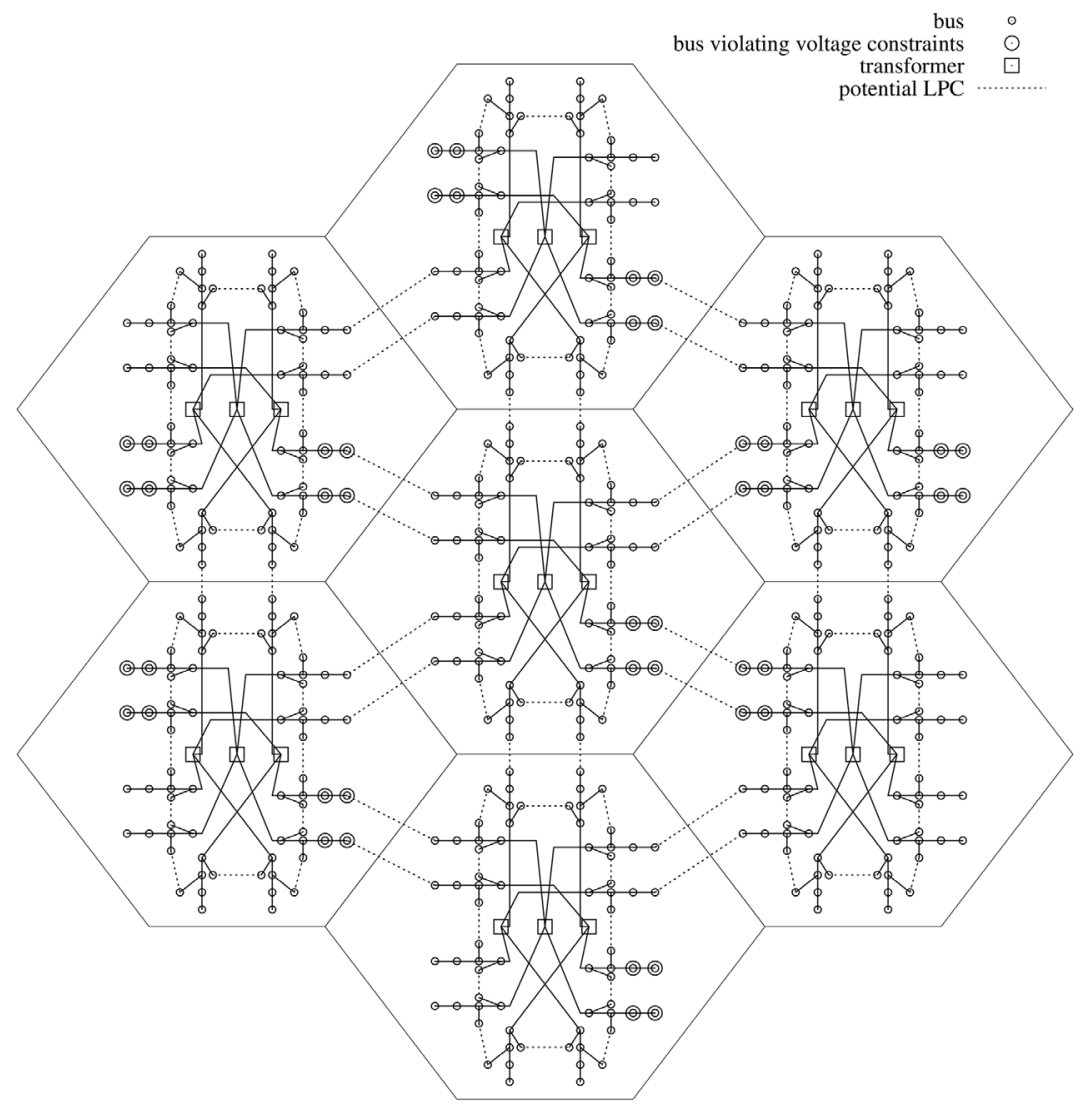

Figure 3 - Power distribution system.

In the calculation, the initial condition was a violation of the operational constraints arising simultaneously in multiple nodes at the ends of the feeders due to a voltage increase (number of nodes where violation occurred: 44), because of the interconnection of the distributed power sources. This initial condition was used to determine the optimal installation location and capacity of the LPC. Simulations were then carried out using the numerical optimizer NUOPT on a $2.00 \mathrm{GHz}$ Xeon E5507 with two processorsand 12.0 GB memory. Examples of modern portfolio optimization problems were given by Scherer \& Martin (2005).

It was shown that the minimum-cost solution in resolving all voltage violations using linear approximation had a calculation time 1/10 that of the previously developed local search method. The results of these calculations are shown in Tables 1 and 2. As can be seen from Table 1 and 2 , our linear approximation approach required OPF to be solved only 5 times, in contrast to the 84 times required when local search was used. 
This reduction in searching is because there is no requirement to search the $0-1$ variable relating to the installation when using the linear approximation method. Linear approximation is a technique that lacks global convergence, and it is not necessary to converge on an optimal solution. In our simulation, the same solution was obtained when using local search and linear approximation, in both cases a suitable solution was obtained for the positional relation with constraint violations in the system.

Although the efficiency of the local search method can be improved by using a set in which the variables representing the installation descision are fixed, the calculation time remains much longer than that of the linear approximation method.

Table 1 - Calculation results (local search).

\begin{tabular}{cccc}
\hline Iterations & $\begin{array}{c}\text { Number } \\
\text { of LPCs }\end{array}$ & Cost & $\begin{array}{c}\text { Number of times } \\
\text { OPF solved }\end{array}$ \\
\hline 0 & 15 & 17.78 & 2 \\
1 & 14 & 16.92 & 20 \\
2 & 13 & 15.99 & 20 \\
3 & 12 & 15.13 & 12 \\
4 & 11 & 13.75 & 16 \\
5 & 11 & 13.75 & 14 \\
\hline \multicolumn{2}{l}{ Total number of times OPF solved } & 84 \\
Calculation time & & $15070(\mathrm{sec})$ \\
\hline
\end{tabular}

Table 2 - Calculation results (linear approximation).

\begin{tabular}{cccc}
\hline Iterations & $\begin{array}{c}\text { Number } \\
\text { of LPCs }\end{array}$ & Cost & $\begin{array}{c}\text { Number of times } \\
\text { OPF solved }\end{array}$ \\
\hline 1 & 16 & 18.48 & 1 \\
2 & 15 & 17.71 & 1 \\
3 & 13 & 15.71 & 1 \\
4 & 11 & 13.75 & 1 \\
5 & 11 & 13.75 & 1 \\
\hline \multicolumn{7}{l}{ Total number of times OPF solved } \\
Calculation time \\
\hline
\end{tabular}

\subsection{Investigation of multi-scenario optimization}

We next investigated multi-scenario optimization by performing a simulation that simultaneously took into consideration two scenarios with different demand patterns, using the same model. In the two scenarios, demand increased on a total of eight buses. In both scenarios, six buses had a demand increase of $2 \mathrm{MW}$, but the locations of buses with a demand increase of $3 \mathrm{MW}$ were different. This is shown in Figures 4, 5 and 6. No account was taken of distributed power sources, and the optimal installation location and capacity of the LPC was determined from an initial 
condition of voltage constraint violations arising in multiple nodes at the ends of feeders (number of nodes where violation occurred: 40). This was due to an increase in demand, with violations of the line thermal capacity constraint arising concurrently in 10 lines. First, we determined the optimal location for the LPC in each individual scenario.

Table 3 - Calculation results (per scenario).

\begin{tabular}{cccc}
\hline Scenario & Solution method & $\begin{array}{c}\text { Calculation } \\
\text { time(s) }\end{array}$ & $\begin{array}{c}\text { Number of } \\
\text { OPF times }\end{array}$ \\
\hline 1 & local search & 4997 & 48 \\
2 & linear approximation & 391 & 4 \\
1 & local search & 3667 & 70 \\
2 & linear approximation & 385 & 4 \\
\hline
\end{tabular}

As can be seen from Table 3, the calculation time when using the linear approximation method was much shorter than that of the local search method. The optimal solutions obtained are shown in Figures 4 and 5.

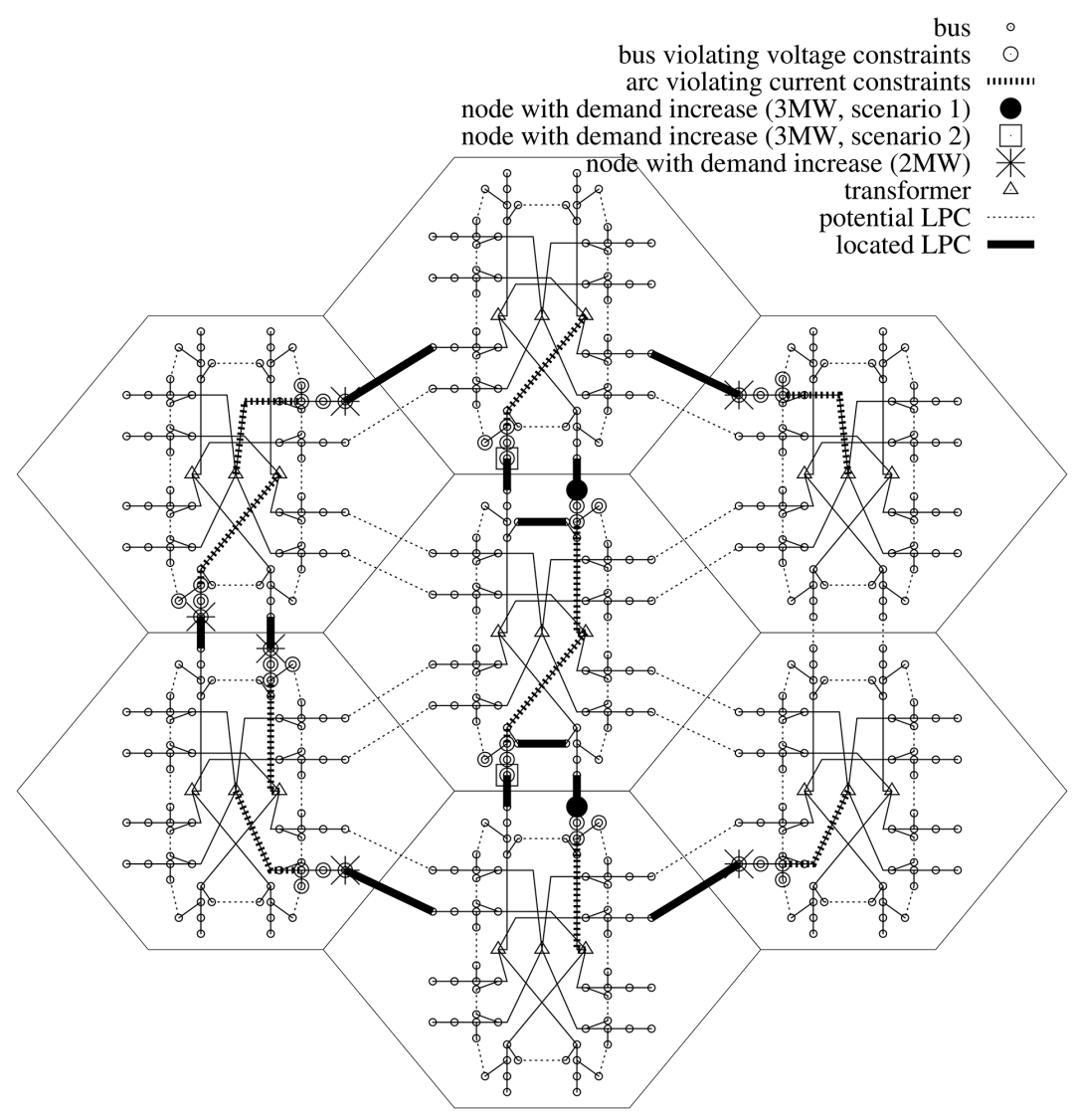

Figure 4 - Optimal location (scenario 1). 


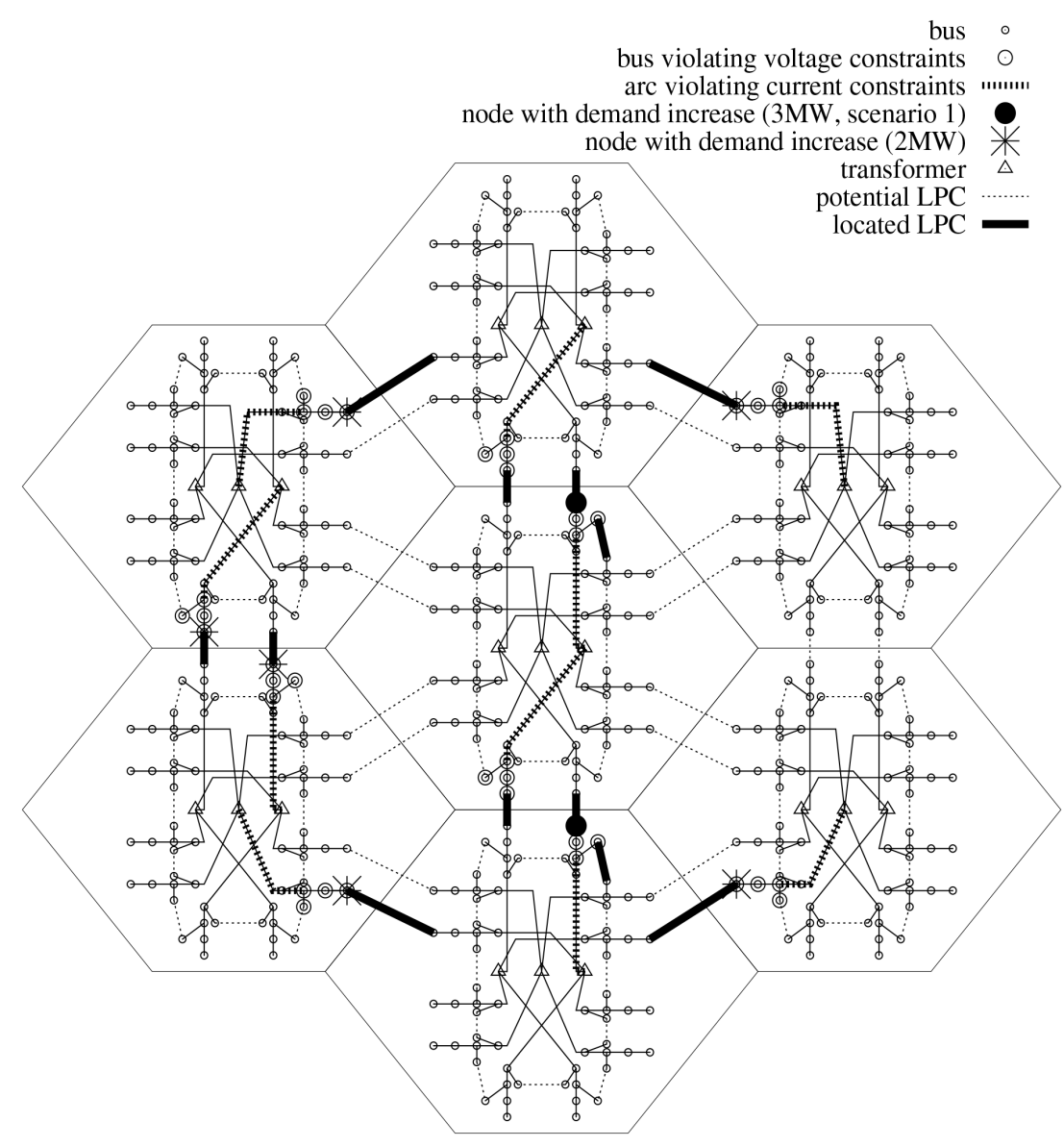

Figure 5 - Optimal location (scenario 2).

Next, we consider a model that takes multiple scenarios simultaneously into account. In this model, the variables $x$ and $y$ are defined as deterministic variables and the variables $e$ and $f$ are defined as stochastic variables representing voltage values. The variables $e$ and $f$ have the subscript $s$ for the scenario $s$, and it can vary for each scenario. The stochastic multi-scenario problem we consider is an extension problem of the original problem (LPC-installation), and it is defined so that the constraints are satisfied for all scenarios. Figure 6 shows the optimal locations when both scenarios were considered.

When the single scenarios were resolved separately, 14 LPC units were installed, whereas when multiple scenarios are considered simultaneously, the number fell to 12 units. As shown in Figure 6, when the scenarios produce different installation patterns, it is possible to reduce the number of units by considering multiple scenarios simultaneously. The calculation results are given in Tables 4 and 5. It can be seen that the LPC installation locations can be determined quickly using the linear approximation method. 


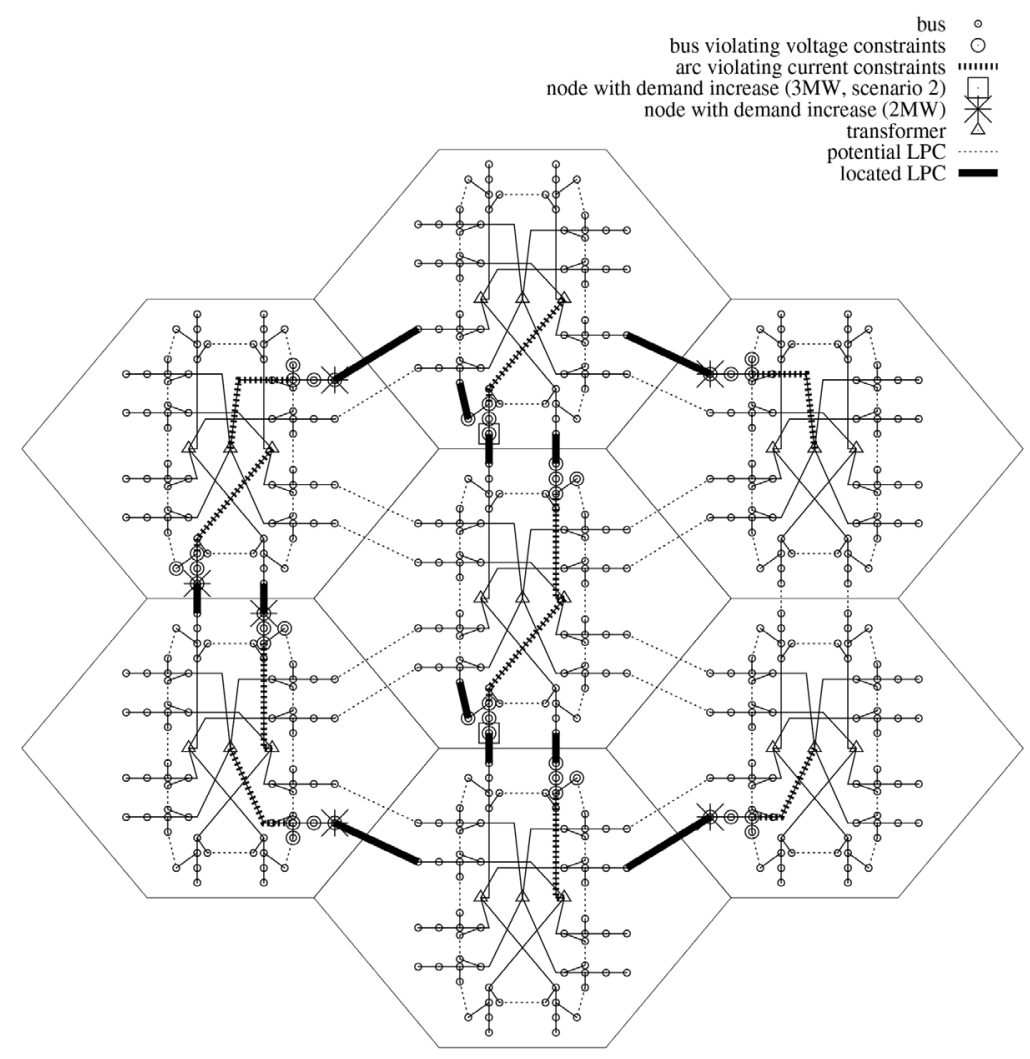

Figure 6 - Optimal location (multi-scenario).

\section{CONCLUSIONS}

When adressing the LPC optimal installation problem, conventional methods require repeated calculations to be performed both when searching for an installation location and for caluculating the OPF. These approaches require long computational time to produce a solution and make it difficult to perform optimization under multi-scenario constraints. In the present study, we developed a more efficient approach for solving practical problems. Using a technique that linearly approximates the installation cost, it becomes possible to obtain solutions through nonlinear optimization of the minimum conditions.

This increases the speed of the process. We investigated the effectiveness of our novel technique using a full-scale system model, and demonstrated its capacity to minimize the cost of resolving all voltage violations efficiently, and with a shorter calculation time than conventional techniques based on local searches.

Our technique was also applied to a problem with multi-scenario flow constraints. When the demand pattern were different in each scenario, our optimization approach was able to resolve the multiple scenarios simultaneously. This new technique has potential applications across a wide range of optimization problems in the electric power filed. 
Table 4 - Calculation results with multiple scenarios (local search).

\begin{tabular}{cccc}
\hline Iterations & $\begin{array}{c}\text { Number } \\
\text { of LPCs }\end{array}$ & Cost & $\begin{array}{c}\text { Number of times } \\
\text { OPF solved }\end{array}$ \\
\hline 0 & 24 & 35.78 & 2 \\
1 & 22 & 33.79 & 2 \\
2 & 21 & 32.86 & 4 \\
3 & 20 & 31.94 & 2 \\
4 & 16 & 27.39 & 4 \\
5 & 16 & 27.38 & 4 \\
6 & 16 & 27.26 & 4 \\
7 & 14 & 25.25 & 4 \\
8 & 13 & 24.25 & 6 \\
9 & 13 & 24.23 & 6 \\
10 & 13 & 24.22 & 6 \\
11 & 12 & 23.27 & 6 \\
12 & 12 & 23.27 & 24 \\
\hline \multicolumn{2}{l}{ Total number of times OPF solved } \\
Calculation time & 74 \\
\hline
\end{tabular}

Table 5 - Calculation results with multiple scenarios (slope scaling).

\begin{tabular}{cccc}
\hline Iterations & $\begin{array}{c}\text { Number } \\
\text { of LPCs }\end{array}$ & Cost & $\begin{array}{c}\text { Number of times } \\
\text { OPF solved }\end{array}$ \\
\hline 1 & 24 & 35.70 & 1 \\
2 & 20 & 31.79 & 1 \\
3 & 16 & 27.37 & 1 \\
4 & 13 & 24.30 & 1 \\
5 & 13 & 24.27 & 1 \\
6 & 12 & 23.27 & 1 \\
\hline \multicolumn{4}{l}{ Total number of times OPF solved } \\
Calculation time \\
\hline
\end{tabular}

\section{REFERENCES}

[1] BAi X, WeI H, FuJisAWA K \& WANG Y. 2008. Semidefinite programming for optimal power flow problems. Electrical Power and Energy Systems, 30: 383-392.

[2] Bazaraa MS, Sherali HF \& Shetty CM. 1993. Nonlinear Programming-Theory and Algorithm, second edition, John Wiley \& Sons, New York.

[3] Kim D \& PARDALOS PM. 1999. A solution approach to the fixed charge network flow problem using a dynamic slope scaling procedure. Operations Research Letters, 24: 195-203.

[4] Kim D \& Pardalos PM. 2000. Dynamic slope scaling and trust interval Techniques for solving concave piecewise linear network flow problems. Networks, 35: 216-222. 
[5] Kim D \& PARDAlos PM. 2000. A dynamic domain contraction algorithm for nonconvex piecewise linear network flow problems. Journal of Global Optimization, 17: 225-234.

[6] Molzahn DK, Holzer JT, Lesieutre BC, DeMarco CL. 2013. Implementation of a LargeScale Optimal Power Flow Solver Based on Semidefinite Programming. IEEE Transactions on Power Systems, 28: 3987-3998.

[7] Ruszczyński A \& Shapiro A. 2003. Stochastic Programming (Handbooks in Operations Research and Management Science, 10), Elsevier.

[8] Scherer B \& MARTin D. 2005. Modern Portfolio Optimization with NUOPT ${ }^{T M}$, S-PLUS®, and $S+$ Bayes $^{T M}$, Springer.

[9] Shapiro A, Dentcheva D \& Ruszczyński A. 2009. Lectures on Stochastic Programming: Modeling and Theory, SIAM.

[10] Shinn T. 1999. Numerical solution technique for joint chance-constrained programming problem An application to electric power capacity expansion. Journal of the Operations Research Society of Japan, 42(2): 128-140.

[11] Shinna T \& BIRGE JR. 2003. Multistage stochastic programming model for electric power capacity expansion problem. Japan Journal of Industrial and Applied Mathematics, 20(3): 379-397

[12] Shina T \& Birge JR. 2004. Stochastic unit commitment problem. International Transactions in Operational Research, 11(1): 19-32.

[13] Shinna T \& Watanabe I. 2004. Lagrangian relaxation method for price-based unit commitment problem. Engineering Optimization, 36(6): 705-719.

[14] Wood AJ \& Wollenberg BJ. 1996. Power Generation. Operation and Control, John Wiley \& Sons, New York.

[15] ZHU J. 2015. Optimization of Power System Operation, Wiley. 